\title{
SOME CONSIDERATIONS REGARDING THE EXACT SOLUTION IN THE ONE PHASE-STEFAN PROBLEM
}

\author{
A. Boucíguez ${ }^{\mathrm{a}}$, ABSTRACT \\ R. Lozano \\ The one phase Stefan problem in a semi - infinite slab with heat flux boundary \\ and M. A. Lara ${ }^{\mathbf{b}}$ condition proportional to $t^{1 / 2}$ and with constant temperature boundary \\ condition are presented here. In these two cases the exact solution exists, the \\ relation between the two boundary conditions is presented here, and the \\ ${ }^{a}$ Facultad de Ciencias Exactas \\ equivalence between the two problems is demostrated. \\ Universidad Nacional de Salta \\ Av. Bolivia 5150 (4400) Salta Argentina \\ Tel: 543874255424 \\ Keywords: phase change materials, Stefan Problem, exact solution, \\ boundary condition \\ bouciga@unsa.edu.ar \\ lozanor@unsa.edu.ar \\ ${ }^{\mathrm{b}}$ Facultad de Ciencias Agrarias \\ Universidad Nacional de Rosario \\ Investigador del CONICET \\ Pellegrini 250 (2000) Rosario Argentina. \\ Tel: 543414495467 int.33 \\ malara@fceia.unr.edu.ar
}

\section{NOMENCLATURE}

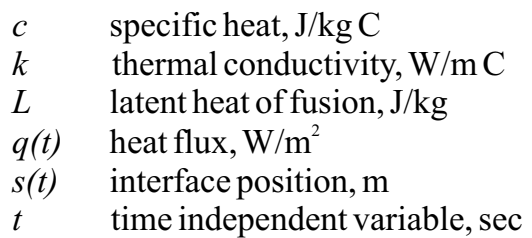

\section{Greek symbols}

$\alpha \quad$ thermal diffusivity, $\mathrm{m}^{2} / \mathrm{sec}$

$\rho \quad$ Density, $\mathrm{kg} / \mathrm{m}^{3}$

\section{INTRODUCTION}

The free boundary problem presents many application in physics and engineering. In particular, it is interesting to study the one called "one phase Stefan problem", (Alexiades and Solomon, 1993) which takes place in a semi - infinite slab when the initial phase is solid (liquid) at the melting point; the material is heated (cooled) from the side and as a consequence the temperature increases (decreases). The substance changes to liquid (solid) phase in the vicinity of the boundary, leaving the rest of the solid (liquid) at the same initial temperature. With time, the liquidified (solidified) zone enlarges itself. For this reason, this problem is named "one phase" because it must be solved only within the region where the phase change takes place. The temperature distribution in this region and the interface position (free boundary) are unknown.
This problem has exact solutions when the condition in the boundary is:

1. Constant temperature

2. Heat flux $q(t)=q_{0} / t^{1 / 2}$

Figure 1 shows a scheme of the phase change material and the boundary condition.

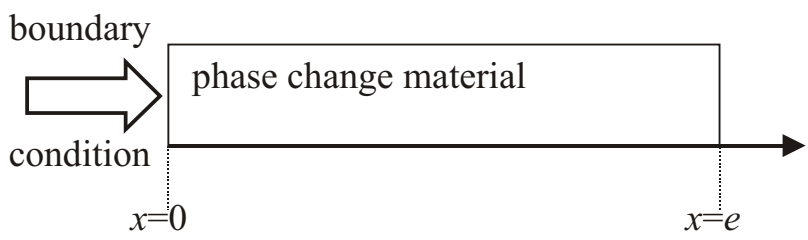

Figure 1: Scheme of the phase change material and the boundary condition.

In a previous work (Lozano et al, 2003) it was observed that when the flux is expressed as $q(t)=q_{0} / t^{1 / 2}$, the temperature in the fixed face stays constant. As Figures 2 to 5 show, for a given $q_{0}$ the temperature in the fixed face is constant during the complete process.

In fact, in Figure 2, the temperature distribution for four different times $(5,1015$ and 20 hours $)$ is shown when $q_{0}$ is $100 \mathrm{Wsec} / 1 / \mathrm{m}^{2}$. Figures 3, 4 and 5 also show the temperature distribution when $q_{0}$ is equal to 500, 1000 and $2000 \mathrm{Wsec} / 1 / \mathrm{m}^{2}$, respectively. The phase change material used for the evaluated temperature is water. For each case the temperature in the fixed face results constant. These Figures might suggest a connection between the two boundary conditions. In this work, this equivalence has been proved, that is to say: one is a consequence of the other and reciprocally. 


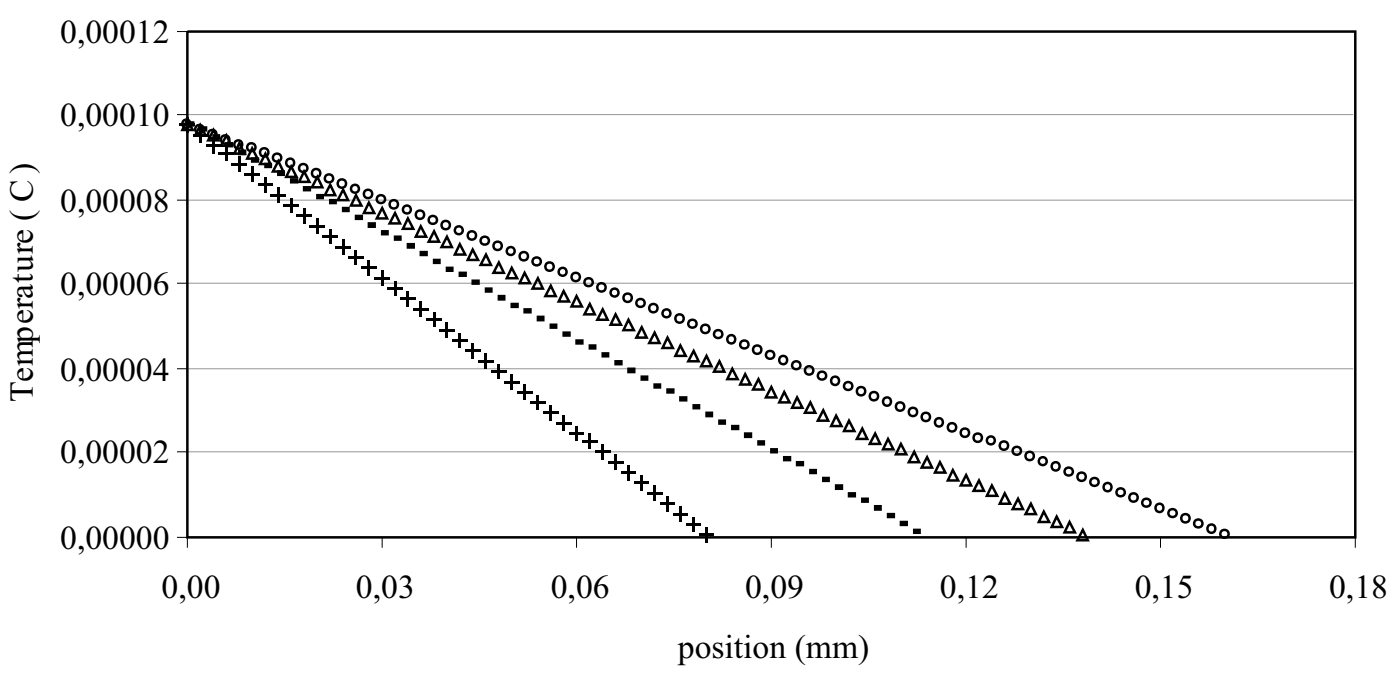

$+5 \mathrm{hs}-10 \mathrm{hs} \Delta 15 \mathrm{hs} \circ 20 \mathrm{hs}$

Figure 2: Temperature distribution in the phase change material, when $q_{0}=100 \mathrm{Wsec} / 1 / \mathrm{m}^{2}$

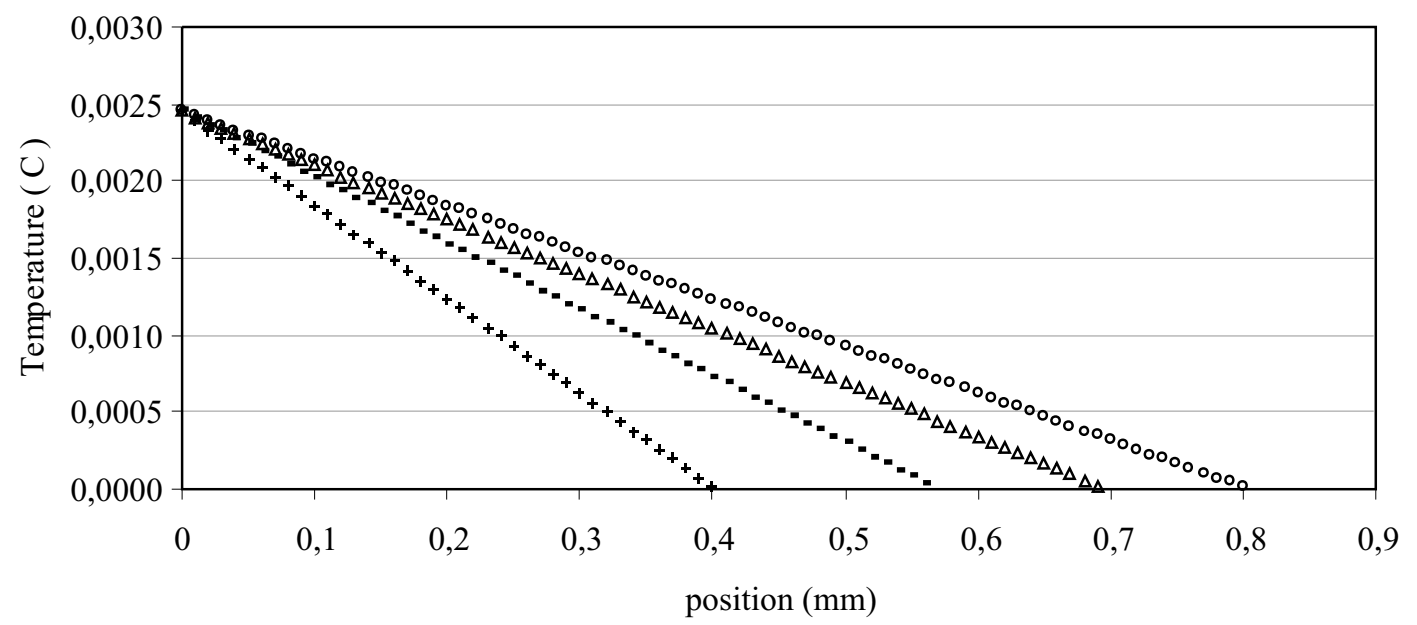

$+5 \mathrm{hs}-10 \mathrm{hs} \Delta 15 \mathrm{hs} \circ 20 \mathrm{hs}$

Figure 3: Temperature distribution in the phase change material, when $q_{0=} 500 \mathrm{Wsec} / 1 / \mathrm{m}^{2}$

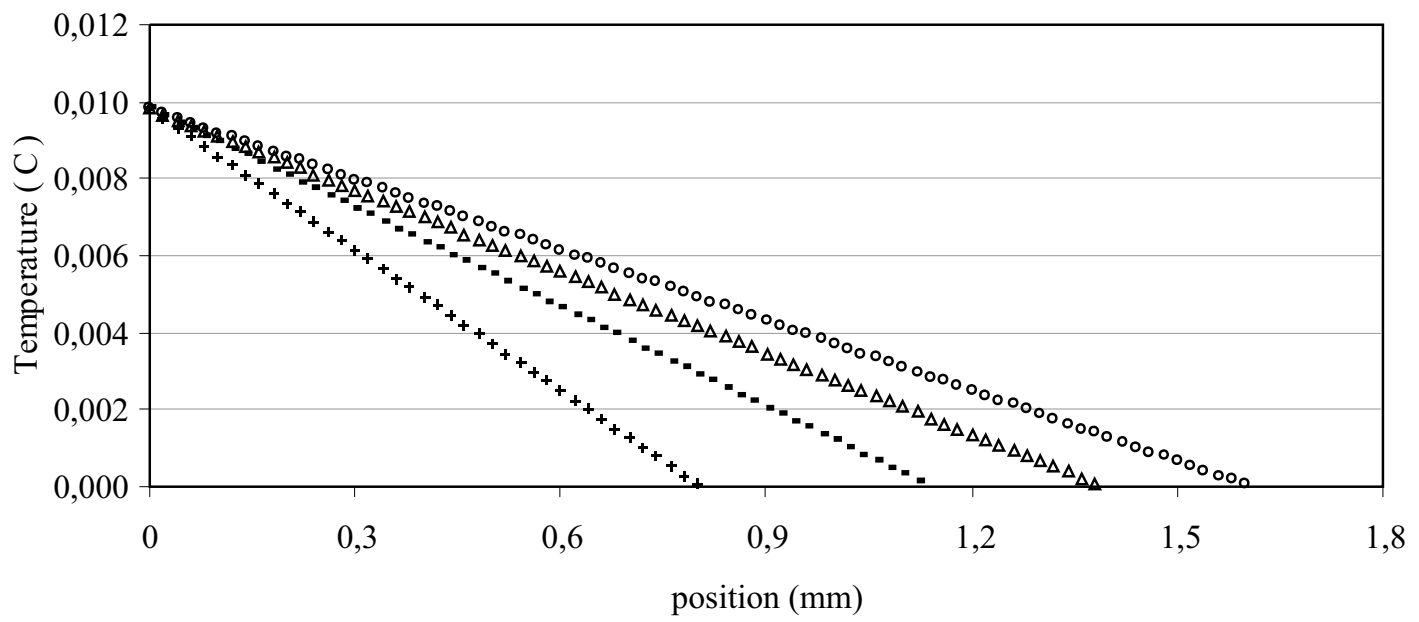

$+5 \mathrm{hs}-10 \mathrm{hs} \Delta 15 \mathrm{hs} \circ 20 \mathrm{hs}$

Figure 4: Temperature distribution in the phase change material, when $q_{0}=1000 \mathrm{Wsec} / 1 / \mathrm{m}^{2}$ 


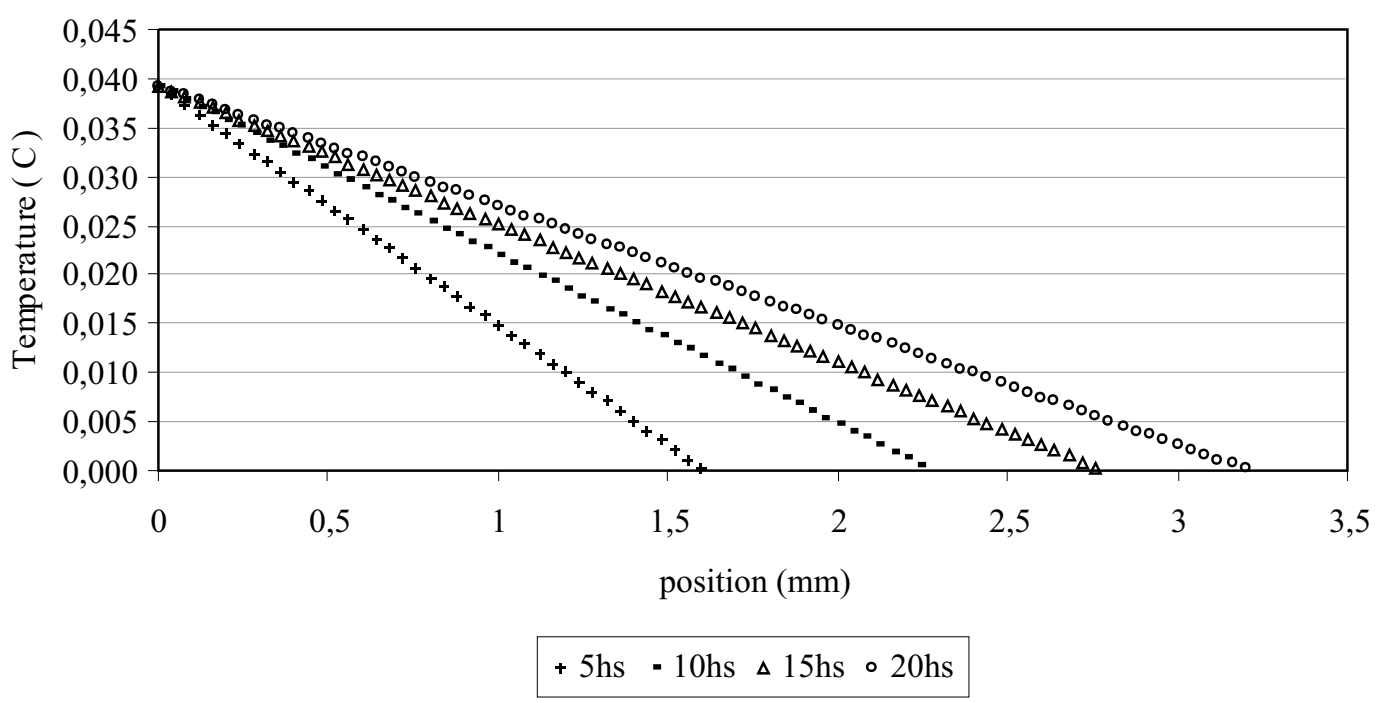

Figure 5: Temperature distribution in the phase change material, when $q_{0=} 2000 \mathrm{Wsec} / 1 / \mathrm{m}^{2}$

\section{MATHEMATICALFORMULATION}

The one phase Stefan problem can be formulated as a heat conduction problem in a semi infinite slab, (Boucíguez, et al 2003) with two kinds of boundary conditions: constant temperature or heat flux proportional to $t^{1 / 2}$.

The problem is completely described by the following equation:

$$
\begin{gathered}
\frac{\partial u}{\partial t}=\alpha \frac{\partial^{2} u}{\partial x^{2}}, 0<t<\tau, 0<x<s(t) \leq \infty \\
u(s(t), t)=0, \forall t>0,0<t<\tau \\
\rho L \frac{d s(t)}{d t}=\mp k \frac{\partial u(x=s(t))}{\partial x} \forall t>0,0<t<\tau \\
u(x, 0)=0,0<x \leq \infty \\
s(0)=0
\end{gathered}
$$

and for the constant temperature case

$u(0, t)=u_{0}, \quad \forall t>0,0<t<\tau, u_{0}>0$

or for the heat flux proportional to $t^{1 / 2}$

$\left.k \frac{\partial u}{\partial x}\right|_{(0, t)}=-\frac{q_{0}}{\sqrt{t}}, \forall t>0,0<t<\tau, q_{0}>0$

Where $\alpha$ is the thermal diffusivity, $k$ is the thermal conductivity, $p$ is the density, $c$ is the specific heat, and $L$ is the latent heat of fusion.

The function $s(t)$, unknown a priori, is the interface position as a function of $t$; and $u(x, t)=T(x, t)-T_{f}$, is the difference between the substance temperature $T(x, t)$ and the fusion temperature $T_{f}$. At the same time $u_{0}=T_{0}-T_{f}$, is the difference between the temperature at the fixed face $T_{0}$ and $T_{f}$.
The minus sign in Eq. 3 represents the case in which the phase change material is initially solid; if the initial condition is liquid the plus sign is applied. This is the Stefan equation; it represents the energy conservation on the interface position.

The Eqs. (6.1) and (6.2), show the two possible boundary conditions: constant temperature (6.1) and heat flux $\sim 1 / t^{1 / 2}(6.2)$. They are the only two cases where this problem has exact solution.

In the two cases, the analytical solution is obtained introducing the similarity variable, (Alexiades and Solomon, 1993), defined by:

$$
\xi=\frac{x}{2 \sqrt{\alpha t}}
$$

The exact solution of the problem is obtained replacing (7) into Eqs. (1) to (6), hence the interface position is:

$$
s(t)=2 \lambda \sqrt{\alpha t}
$$

Where $\lambda$ is a parameter to be determined for each case. For the constant temperature boundary condition $\lambda$ is given by the equation

$$
\lambda \cdot \operatorname{erf}(\lambda) \cdot \exp \left(\lambda^{2}\right)=\frac{\text { Ste }}{\sqrt{\pi}}
$$

For the flux boundary condition is given by the equation

$$
\lambda \cdot \exp \left(\lambda^{2}\right)=\frac{1}{\rho L} \frac{q_{0}}{\sqrt{\alpha}}
$$

where Ste $=\frac{c}{L} u_{0}$, is the Stefan number.

The two Eqs. (9.1) and (9.2) are transcendental and its resolution allows obtaining the exact solution for each problem. The solution of these equations is unique and it implies the uniqueness of the similarity solution. In consequence, the Stefan problem admits only one solution. 
The temperature distribution is given by:

1) For the temperature boundary condition

$$
u(x, t)=u_{0}-u_{0} \cdot \frac{\sqrt{\pi}}{\text { Ste }} \cdot\left(\frac{f(\xi)}{f(\lambda)}\right)
$$

2) For the flux boundary condition

$$
u(x, t)=\frac{q_{0}}{k} \cdot \sqrt{\alpha \cdot \pi}[f(\lambda)-f(\xi)]
$$

where $f()$ denotes the error function,

$$
f(\xi)=\operatorname{erf}(\xi)=\frac{2}{\sqrt{\pi}} \int_{0}^{\xi} \exp \left(-v^{2}\right) \cdot d v
$$

The basic properties of the error function are:

$$
\left\{\begin{array}{l}
f(0)=0 ; f(\infty)=1 ; f(-\xi)=-f(\xi) \\
\frac{d f}{d \xi}=f^{\prime}(\xi)=\frac{2}{\sqrt{\pi}} \exp \left(-\xi^{2}\right)>0 \\
\frac{d^{2} f}{d \xi^{2}}=f^{\prime \prime}(\xi)=-2 \xi f^{\prime}(\xi)=-\frac{4 \xi}{\sqrt{\pi}} \exp \left(-\xi^{2}\right)
\end{array}\right.
$$

\section{RELATIONS BETWEEN THE TWO BOUNDARY CONDITIONS}

It is useful to name the parameter in Eqs. (9) and (10), as $_{1}$ for the temperature condition (case 1) and ${ }_{2}$, for the flux condition (case 2);

$$
\begin{gathered}
\lambda_{1} \cdot \operatorname{erf}\left(\lambda_{1}\right) \cdot \exp \left(\lambda_{1}^{2}\right)=\frac{\text { Ste }}{\sqrt{\pi}} \\
\lambda_{2} \cdot \exp \left(\lambda_{2}^{2}\right)=\frac{1}{\rho L} \frac{q_{0}}{\sqrt{\alpha}}
\end{gathered}
$$

and in consequence, Eq. (10) becomes:

$$
\begin{aligned}
& u(x, t)=u_{0}\left[1-\left(\frac{f(\xi)}{f\left(\lambda_{1}\right)}\right)\right] \\
& u(x, t)=\frac{q_{0}}{k} \cdot \sqrt{\alpha \cdot \pi} \cdot\left[f\left(\lambda_{2}\right)-f(\xi)\right]
\end{aligned}
$$

The equivalence between the two cases will be proved. Evaluating $u(0, t)$ from Eq. (10.2):

$u(0, t)=\frac{q_{0}}{k} \sqrt{\alpha \pi} f\left(\lambda_{2}\right)=\frac{q_{0}}{k} \sqrt{\alpha \pi} \operatorname{erf}\left(\lambda_{2}\right)=c t e$.

from Eq. (9.1), $\left.f()_{1}\right)$ results

$f\left(\lambda_{1}\right)=\operatorname{erf}\left(\lambda_{1}\right)=\frac{\text { Ste }}{\sqrt{\pi}} \cdot \frac{1}{\lambda_{1} \exp \left(\lambda_{1}^{2}\right)}$

replacing $f\left({ }_{1}\right)$ in Eq. (10.1)

$$
u(x, t)=u_{0} \cdot\left[1-\frac{\sqrt{\pi}}{\text { Ste }} \lambda_{1} \cdot \exp \left(\lambda_{1}^{2}\right) f(\xi)\right]
$$

Taking the derivative of this expression with respect to $x$, and in virtue of the properties of the error function, it yields

$$
\begin{aligned}
& u_{x}(x, t)=u_{0}\left[-\lambda_{1} \cdot \exp \left(\lambda_{1}^{2}\right) \frac{\sqrt{\pi}}{\text { Ste }} \cdot \frac{f^{\prime}(\xi)}{2 \sqrt{\alpha t}}\right]= \\
& =-u_{0} \frac{1}{\text { Ste }} \cdot \frac{1}{\sqrt{\alpha t}} \lambda_{1} \cdot \exp \left(\lambda_{1}^{2}\right) \exp \left(-\xi^{2}\right)
\end{aligned}
$$

The heat flux at the boundary is $q(t)=k u_{x}(0, t)$, hence evaluating expression (16) at $x=0$, the heat flux is:

$$
q(t)=-k u_{x}(0, t)=\left(\frac{k u_{0}}{S t e}\right) \frac{1}{\sqrt{\alpha t}} \lambda_{1} \exp \left(\lambda_{1}^{2}\right)
$$

Expressing this equation as:

$$
q(t)=\frac{q_{1}}{\sqrt{t}}
$$

where

$q_{1}=\frac{k u_{0}}{\text { Ste }} \frac{1}{\sqrt{\alpha}} \lambda_{1} \exp \left(\lambda_{1}^{2}\right)=\rho L \sqrt{\alpha} \lambda_{1} \exp \left(\lambda_{1}^{2}\right)$

replacing in Eq. (17) yields

$-k u_{x}(0, t)=q(t)=\frac{q_{1}}{\sqrt{t}}=\frac{\rho L \sqrt{\alpha}}{\sqrt{t}} \lambda_{1} \exp \left(\lambda_{1}^{2}\right)$

This expression is similar to Eq. (6.2). The comparison of the boundary condition $u_{0}(6.1)$ with the solution at the boundary for the flux case $u(0, t)(13)$ shows that they are equal if:

$$
u_{0}=\frac{q_{0}}{k} \sqrt{\alpha \pi} \cdot \operatorname{erf}\left(\lambda_{2}\right)
$$

The comparison of the boundary condition for the flux case, Eq. (6.2), with the solution in the boundary for the temperature case, Eq. (18), shows that they are equal if:

$$
-k u_{x}(0, t)=\frac{q_{0}}{\sqrt{t}}=\frac{\rho L \sqrt{\alpha}}{\sqrt{t}} \lambda_{2} \exp \left(\lambda_{2}^{2}\right)
$$

Equations (20) and (22), are the same if and only if ${ }_{1}={ }_{2}=$. In this case:

$$
q_{1}=q_{0}=\rho \cdot L \cdot \sqrt{\alpha} \cdot \lambda \cdot \exp \left(\lambda^{2}\right)
$$

and replacing this value in the Eq. (21)

$$
\begin{aligned}
& u_{0}=\frac{\rho \cdot L \cdot \sqrt{\alpha}}{k} \cdot \sqrt{\alpha \pi} \cdot \lambda \cdot \operatorname{erf}(\lambda) \cdot \exp \left(\lambda^{2}\right)= \\
& =\frac{\sqrt{\pi}}{\text { Ste }} \cdot u_{0} \cdot \lambda \cdot \operatorname{erf}(\lambda) \cdot \exp \left(\lambda^{2}\right)
\end{aligned}
$$


Dividing both sides of this equation by $u_{0}$ /Ste, we obtain Eq. (9.1). Then it is proved that the two boundary conditions are not independent: one is a consequence of the other and reciprocally.

\section{NUMERICAL EVALUATION}

A simple numerical evaluation for the situations discussed in the previous section is presented here, for different values $q_{0}$. The results are presented in Table 1; the second column is the value of corresponding to Eq. (9.2), that is to say ${ }_{2}$. The third column is the obtained temperature using this value and Eq. (10.2). The fourth column is the Stefan number evaluated with this last temperature and finally the fifth column is the value of corresponding to Eq. (9.1), that is to say ${ }_{1}$, evaluated with the Stefan number of column four. All these calculations where made using the Mathematical Support of the Scientific Work Place, so the precision is assured.

Table 1: Values of ${ }_{1},{ }_{2}$, temperatures and Stefan numbers for different values of $q_{0}$

\begin{tabular}{|c|c|c|c|c|}
\hline$q_{0}$ & $\lambda_{2}$ & $u(0, t)$ & Ste & $\lambda_{1}$ \\
\hline 100 & 0,00078215 & 0,0000981035 & $1,223510^{6}$ & $7,821510^{4}$ \\
\hline 500 & 0,0039107 & 0,00215254 & 0,0000305872 & $3,910710^{3}$ \\
\hline 1000 & 0,0078210 & 0,00980956 & 0,000122341 & $7,82110^{3}$ \\
\hline 2000 & 0,0156391 & 0,0392285 & 0,000489244 & $1,563910^{2}$ \\
\hline 3000 & 0,0234515 & 0,0882283 & 0,00110035 & $2,345210^{2}$ \\
\hline 4000 & 0,0312554 & 0,156761 & 0,00195507 & $3,125510^{2}$ \\
\hline 5000 & 0,0390478 & 0.244761 & 0,00305257 & $3,904810^{2}$ \\
\hline
\end{tabular}

\section{DISCUSSION AND CONCLUSION}

The analysis of Figures 2 to 5, for a particular material (water), shows that the heat flux $\mathrm{q}_{0} / t^{1 / 2}$ produces a constant temperature in the fixed face.

The obtained results are summarized in Table 2. This table also shows that when $q_{0}$ increases, the temperature in the fixed face is multiplied by the squared ratio between the new and the old $q_{0}$. In this table the ratio was obtained taking 100 , as the first value. So, for $q_{0}=500$, this factor is $500 / 100=5$, for $q_{0}=1000$, is $1000 / 100=10$ and so on. As this ratio is only a consequence of $q_{0}$, because for a given material, this is the only factor that changes in Eq. (10.2), this relation is also valid for all substances.

Table 2: Temperature in the fixed face for different $q_{0}$

\begin{tabular}{|c|c|c|c|}
\hline$q_{0}\left(\mathrm{Wsec}^{1 / 2} / \mathrm{m}^{2}\right)$ & $u(0, t)(\mathrm{C})$ & Incremental factor & Resultant temperature \\
\hline 100 & 0,00010 & 1 & $0,00010 * 1$ \\
\hline 500 & 0,0025 & $5^{2}=25$ & $0,00010 * 25$ \\
\hline 1000 & 0,010 & $10^{2}=100$ & $0,00010 * 100$ \\
\hline 2000 & 0,04 & $20^{2}=400$ & $0,00010 * 400$ \\
\hline
\end{tabular}

The analysis presented in the previous section shows that the equivalence between the two boundary conditions is true for any phase change material, such as the numerical evaluation shows at a first instance.

Consequently, it is proved that the two well known analytical solutions of the one phase Stefan problem, (one for the temperature boundary condition, the other for the heat flux condition) are not independent. If one of them is imposed, the other one is satisfied.

\section{ACKNOWLEDGEMENT}

The authors are grateful to Dr. Luis Cardón for the revision of this article and to Dra. Marta Pocoví for the English revision. This paper has been financed by Investigation Project $\mathrm{N}^{\mathrm{o}}$ 1264, CIUNSa. National University of Salta, Argentina.

\section{REFERENCES}

Alexiades, V and Solomon, A. 1993. Mathematical Modeling of Melting and Freezing Processes. Hemispher Publishing Corporation.

Boucíguez, A; Villa, L and Lara, M. 2003. Thermal Conditioning Using Phase Change Materials. Engenharia Termica, $\mathrm{N}^{\mathrm{o}} 3$, p.71 74 .

Lozano, R; Boucíguez, A; Lara, M and Villa, L. 2003. Comportamiento de la frontera Libre en Materiales de Cambio de Fase con Condiciones de Flujo de Calor Constante. Revista Energías Renovables y Medio Ambiente, Vol. 12,p. 4953. 